\title{
Interpersonal Relationships in Diabetes: Views and Experience \\ of People with Diabetes, Informal Carers, and Healthcare \\ Professionals in Portugal
}

\author{
Relações Interpessoais na Diabetes: Perspetiva e Experiência de \\ Pessoas com Diabetes, Cuidadores Informais, e Profissionais de \\ Saúde em Portugal
}

\author{
Dulce NASCIMENTO DO Ó $\rrbracket^{1}$, Lurdes SERRABULHO ${ }^{1}$, Rogério T. RIBEIRO ${ }^{1,2,3}$, Sónia SILVA ${ }^{1}$, Ana COVINHAS $^{1}$, \\ Maria João AFONSO ${ }^{1}$, José Manuel BOAVIDA ${ }^{1}$, João Filipe RAPOSO ${ }^{1,2}$
}

Acta Med Port 2022 Oct;35(10):729-737 - https://doi.org/10.20344/amp.16817

\section{ABSTRACT}

Introduction: The increasing burden of diabetes poses a great challenge to healthcare systems and economy worldwide. Although modern therapeutic strategies for diabetes are widely available, most patients still fail to achieve optimal clinical targets and well-being. The primary objective of this study was to assess and explore potential drivers and successful management of diabetes among people with diabetes, family members and healthcare professionals in Portugal, by applying the protocol of the multinational study "Diabetes, Attitudes, Wishes and Needs (DAWN2)".

Material and Methods: A total of 767 adults, including 417 people with diabetes, 123 family members and 227 healthcare professionals, participated in the study. Surveys assessed health-related quality of life, self-management, attitudes/beliefs, social support and priorities for improvement areas in diabetes care.

Results: Diabetes has a negative impact on the physical health and emotional well-being of patients in Portugal and is also a psychological burden for family members. Earlier diagnosis and treatment of diabetes were mentioned as a major area of improvement. Healthcare professionals indicated the need for diabetes self-management education.

Conclusion: We have used for the first time in Portugal the DAWN2 protocol to address the wishes, needs, and attitudes of Portuguese diabetes patients, their relatives, and healthcare professionals regarding the disease.

Keywords: Diabetes Mellitus; Portugal; Quality of Life; Self-Management; Social Support

\section{RESUMO}

Introdução: Os encargos crescentes com a diabetes representam um desafio para os sistemas de saúde e economia a nível mundial. Apesar de terapias modernas para a diabetes disponíveis, a maioria das pessoas continua privada de cuidados e bem-estar adequados. O objetivo primário deste estudo foi avaliar e explorar os fatores relevantes para o controlo ativo e eficaz da diabetes para as pessoas com diabetes, familiares e profissionais de saúde em Portugal, aplicando o protocolo do estudo multinacional "Diabetes, Attitudes, Wishes and Needs (DAWN2)".

Material e Métodos: Participaram no estudo 767 adultos (417 pessoas com diabetes, 123 familiares e 227 profissionais de saúde). Foram avaliados a qualidade de vida associada à saúde, autogestão, atitudes/crenças, apoio social e prioridades em áreas de meIhoria no tratamento da diabetes.

Resultados: A diabetes tem um impacto negativo na saúde física e no bem-estar emocional das pessoas em Portugal, sendo também uma carga psicológica para os seus familiares. O diagnóstico e tratamento precoces da diabetes foram indicados como a principal área de melhoria. Profissionais de saúde indicaram a necessidade de educação para a autogestão da diabetes.

Conclusão: Pela primeira vez em Portugal usámos o protocolo DAWN2 para ir ao encontro dos desejos, necessidades e atitudes dos doentes Portugueses com diabetes, os seus familiares e profissionais de saúde relativamente à doença.

Palavras-chave: Apoio Social; Autogestão; Diabetes Mellitus; Portugal; Qualidade de Vida

\section{INTRODUCTION}

Diabetes mellitus (DM) is a complex chronic disease that requires continuous medical care. ${ }^{1}$ Over the past few decades, ageing and unhealthy lifestyles have been contributing to the increased prevalence of type 2 diabetes (T2D), posing an growing challenge to healthcare systems and national economies..$^{2,3}$ In order to prevent or delay DM shortand long-term complications, it is necessary to implement an extensive self-management program of the disease, which should include the adoption of healthy food habits, physical activity, monitoring of blood glucose levels and, if necessary, compliance with medical therapy. $2,4,5$

People with diabetes (PWD) often describe their experiences of managing the disease as emotionally, physically and socially challenging. ${ }^{6}$ Family members may have an active and very important role in supporting and caring for people with diabetes, contributing to patient compliance with treatment and promoting the change and maintenance of a healthy life style in terms of diet and physical

1. APDP - Diabetes Portugal. Lisbon. Portugal.

2. CEDOC - Centro de Estudos de Doenças Crónicas. NOVA Medical School. Lisbon. Portugal.

3. Department of Medical Sciences. University of Aveiro. Aveiro. Portugal.

$\triangle$ Autor correspondente: Dulce Nascimento do Ó. dulce.o@apdp.pt

Recebido/Received: 21/07/2021 - Aceite/Accepted: 24/01/2022 - Publicado Online/Published Online: 03/03/2022 - Publicado/Published: 03/10/2022 Copyright $\odot$ Ordem dos Médicos 2022 
activity. ${ }^{7,8}$ Health care providers (HCPs) are also an important element of the patient support network, by playing a significant role in encouraging patients to improve their quality of life. ${ }^{9}$ Nevertheless, despite all psychosocial and educational support programs for PWD implemented over the last decade, many patients still do not have access to adequate care and support. ${ }^{10}$

The Diabetes Attitudes, Wishes and Needs 2 (DAWN2), is a multinational, interdisciplinary and multi-stakeholder study, aiming to assess and explore potential drivers for active and successful diabetes management among people with diabetes, family members, and healthcare professionals, in response to growing pressure for more cost-effective models of diabetes care. ${ }^{10}$ The DAWN2 protocol is based on three quantitative surveys which explore the experiences and unmet needs of PWD, family members of PWD and healthcare professionals treating PWD. The aim of the study was to generate insights that can promote the development of innovative efforts by all stakeholders to improve the self-management and psychosocial support of people with diabetes. Data from 17 countries using the DAWN2 protocol has been published and areas for improvement as well as best practices were identified that can be used to drive the changes that improve the PWD outcomes. ${ }^{11}$

Here, we report the application of the DAWN2 protocol to the Portuguese setting. We aim to identify unmet needs and benchmark our insights with data from other countries, so that we can contribute to the specific for the Portuguese context for improvement of PWD management and support.

\section{MATERIAL AND METHODS \\ Participants}

The composition of the study population was conceived to portray in a representative manner the population of adults diagnosed with diabetes and their families in Portugal, according to the DAWN2 protocol. ${ }^{10}$ All relevant healthcare professionals - endocrinologists, internal medicine specialists, primary care physician (PCP) / general practitioners (GPs) / family physicians (FPs), nurses, dietitians, etc. - were included, in order to provide an integrated view of the healthcare provided to the population with diabetes in Portugal.

A total of 767 participants were included in the study: 417 PWD, 123 family members/carers (FMs) and 227 HCPs. PWD were divided into type 1 DM patients $(n=89)$ and type 2 DM patients $(n=238)$, of which 170 were noninsulin treated and 158 were insulin treated. FMs were divided into insulin treated relative $(n=86)$ and non-insulin treated relative $(n=37)$. HCPs were divided into primary care physicians / GPs / FPs ( $n=68)$, endocrinologists / internal medicine specialists $(n=40)$ and NDO's - nurses, dietitians and other HCPs $(n=119)$.

The inclusion criteria for the PWD group were diagnosis of diabetes (type 1 or type 2) and for the FMs group was to be involved in the daily care of an adult with type 1 or type 2 diabetes. The inclusion criteria for the HCP group were as follows: PCP/GP/FP providing care for five or more adults with diabetes per month; endocrinologists or internal medicine specialists providing care for 50 or more adults with diabetes per month, and prescribing oral medication, insulin or other injectable medication for diabetes. The inclusion criteria for NDOs were to be general practice/diabetes nurses, dietitians, psychologists or other healthcare professionals, providing care for five or more adults with diabetes per month. All study participants were adults (18 years old or more), were living in Portugal and agreed to participate in the survey upon review of the provided informed consent form.

Subjects diagnosed with diabetes for less than 12 months before the recruitment were excluded, as well as participants with gestational diabetes or without verbal or writing comprehension ability.

The sample source differs between groups of participants, but a convenience sampling method was adopted, using social events and platforms from the Portuguese Diabetes Association (APDP). The duration of the interviews was 40 to 60 minutes, depending on the specific group. Onsite recruitment was adopted in all cases, except for the HCP group, where some participants were recruited by email.

\section{Setting and study design}

This was a cross-sectional study, aiming to achieve a reliable description of the psychosocial factors involving adults with diabetes, their families and healthcare professionals in Portugal. Data from the PWD and FMs groups was collected between January and July 2017. Data from HCPs were collected between January 2017 and April 2018. For the PWD group, 76 telephone and 341 face-to-face interviews were conducted. For the FMs survey, 53 telephone and 70 face-to-face interviews were conducted. In the HCPs group, 227 answered the surveys online, in a self-reported manner. All interviews were conducted in European Portuguese. The name of the sponsor of the study was not disclosed in any of the survey materials.

\section{Data sources}

Three separate survey questionnaires, one for each of the three target study groups (i.e. PWD, FMs, HCPs) were developed. The survey questionnaires incorporated items from the original DAWN study ${ }^{12}$ and new questions, including open-ended items developed by a multidisciplinary, multinational team. ${ }^{10}$ The surveys included standardized scoring scales - the abbreviated version of WHO quality of life assessment questionnaire - (WHOQOL-BREF); EuroQol (EQ-5D); WHO Well-Being index (WHO-5); the Problem Areas in Diabetes scale - short form (PAID-5); the Health Care Climate questionnaire (HCCC); the Summary of Diabetes Self-Care Activities measure (SDSCA) - and also new questions adapted from existing validated instruments - the Diabetes Empowerment scale-short form (DES-SF), Patient Assessment of Chronic Illness Care (PACIC), and Diabetes Family Behaviour checklist (DFBC).

The scoring scales were translated into Portuguese. 
The translations were reviewed by a panel of Portuguese diabetes experts. Selected questions intended for scientific benchmarking were back translated into English by a third independent professional translator and an harmonization review was undertaken, involving the approval of academic experts and of the original authors of the scientific scales.

\section{Statistical analysis}

The responses from participants who completed the survey on face-to-face or telephone interviews were entered in the online survey program by interviewers, using a unique survey link for each participant. Statistical analysis was performed using the Statistical Package for the Social Sciences Software (SPSS 24.0 ${ }^{\circledR}$ ). Descriptive statistics were calculated for all the study variables, including frequencies, percentages, mean and standard deviation, as applicable. For the analysis of PWD data, Type 2 DM patients were analysed as a whole $(n=328)$, and also in two subgroups non-insulin treated $(n=170)$ and insulin treated $(n=158)$.

\section{Ethical considerations}

The DAWN2 study was conducted in accordance with $\mathrm{ICH}-\mathrm{GCP}$, the Helsinki Declaration and national legislation and was submitted and obtained favourable opinion from the local APDP Ethics Committee before implementation. Those interested in participating were requested to provide informed written consent.

\section{Confidentiality}

All data was collected anonymously and there was no way to relate the completed surveys with the participants. The DAWN2 study was submitted and approved by the Portuguese Data Protection Authority (CNPD - Comissão Nacional de Proteção de Dados) before implementation. Approval number 12008/2016, dated 09/November/2016.

\section{RESULTS}

\section{Demographic characterization}

Table 1 summarizes the characteristics of PWD group. In both type 1 and type 2 groups, most subjects were male (51.7\%, $n=46$ and 55.8\%, $n=183$, respectively). Type 1 PWD were mostly aged from 18 to 59 years old $(85,4 \%)$, whereas type 2 PWD were mostly 60 years old or more (76.8\%). On average, type 1 PWD were diagnosed at a younger age than type 2 PWD (22.9 and 48.6 years old, respectively). Most type 1 PWD (70\%) reported to work fulltime, whereas most type 2 PWD (63\%) were retired. Most type 1 and type 2 PWD (55\% and 64\%, respectively) reported a monthly household income between $€ 506$ and $€ 2000$ (low / low-middle class); and $25 \%$ and 15\%, respectively, an income between $€ 2001$ and $€ 5000$ (middle class). The highest education level most frequently reported by type 1 PWD were bachelor's / master's degree (44\%) and secondary education (27\%); whereas among type 2 PWD $43 \%$ had only finished the first cycle of the basic education, $18 \%$ the secondary education, $17 \%$ a bachelor's / master's degree, and $15 \%$ the third cycle of basic education.
FMs were divided into two groups: group $1(n=85)$, whose relatives are insulin treated, and group $2(n=35)$, whose relatives are non-insulin treated. FMs from both group 1 and group 2 were mainly female $(73.3 \%, n=63$ and $83.8 \%, \mathrm{n}=31$, respectively) and the patient's spouse/ partner (group $1-60.5 \%, n=52$; group $2-64.9 \%, n=24$ ). On average, FMs age on group 1 was 56.4 years old, and on group 2, 63.1 years old. From the FMs on group 1, 48\% reported to work full-time and $38 \%$ were retired, whereas $43 \%$ of FMs on group 2 were retired and $24 \%$ worked fulltime. Of note, $2 \%$ of FMs on group 1 reported not working full-time because of PWD condition. Most FMs of group 1 and group 2 (64\% and $62 \%$, respectively) reported a monthly household income between $€ 506$ and $€ 2000$ (low / lowmiddle class); and $18 \%$ and $8 \%$, respectively, an income between $€ 2001$ and $€ 5000$ (middle class). The highest education level most frequently reported by FMs on group 1 were secondary education (27\%), bachelor's / master's degree $(21 \%)$, first cycle of basic education $(21 \%)$, and the third cycle of basic education (17\%). The highest education level most frequently reported by FMs on group 2 were first cycle of basic education (38\%), bachelor's / master's degree $(25 \%)$, and secondary education (16\%).

HCPs were divided into three groups: PCPs/GPs/FPs ( $n=68)$; hospital based specialists $(n=40)$; and nurses/ dietitians/other HCPs (NDOs; $n=119$ ). hospital based specialists were either internal medicine physicians $(45.0 \%, n$ $=18$ ) or endocrinologists $(55.0 \%, n=22)$. All three HCPs groups followed mostly type $2 \mathrm{DM}$ patients: PCPs/GPs 95.6\%; hospital-based specialists - $80 \%$; and NDOs - $89 \%$.

\section{Living with diabetes: the perspective of the person with diabetes}

Table 2 summarizes the data regarding the perceived quality of life (QoL) of PWD. Most type 1 DM patients perceived their QoL as 'Good/Very Good' (67.4\%), whereas type 2 patients perceived their QoL mostly as 'Neither poor nor good' (48.5\%). The perceived QoL of type 2 patients non-insulin treated reported as 'good/very good' was better than the one reported by type 2 insulin treated patients: $48.2 \%$ vs $39.2 \%$. This observation was confirmed by the PAID-5 assessment scale: $50.9 \%$ of type 2 non-insulin treated patients reported 'high distress' versus $72.9 \%$ of type 2 insulin-treated patients.

Table 3 summarizes the impact of diabetes on PWD's QoL in six dimensions: physical health, financial situation, personal relationships, leisure activities, work or study, and emotional well-being. Physical health and emotional wellbeing were the most negatively impacted areas in PWD's QoL for both type 1 and type 2 patients.

PWD attitudes about diabetes were also analysed. For the sentence "I am very worried about the risk of hypoglycaemic events", type 1 patients reported an agreement rating of $61.8 \%(n=55)$ and type $2,48.4 \%(n=158)$. Particularly, hypoglycaemic events at night registered an agreement rating of $68.5 \%(n=61)$ for type 1 and $37.0 \%(n=$ 11) for type 2 . Regarding the sentence "I feel very anxious 
Table 1 - PWD sample characterization $(n=745)$

\begin{tabular}{|c|c|c|c|c|c|}
\hline & & $\begin{array}{l}\text { Type } 1 \\
(n=89)\end{array}$ & $\begin{array}{c}\text { Type } 2 \\
(n=328)\end{array}$ & $\begin{array}{c}\text { Type } 2 \\
\text { non-insulin treated } \\
(n=170)\end{array}$ & $\begin{array}{c}\text { Type } 2 \\
\text { insulin treated } \\
(n=158)\end{array}$ \\
\hline \multirow{2}{*}{ Gender } & Male & $46(51.7 \%)$ & $183(55.8 \%)$ & $106(62.4 \%)$ & $77(48.7 \%)$ \\
\hline & Female & $43(48.3 \%)$ & $145(44.2 \%)$ & $64(37.6 \%)$ & $81(51.3 \%)$ \\
\hline \multirow{3}{*}{ Age } & $18-39$ & $34(38.2 \%)$ & $2(0.6 \%)$ & $0(0.0 \%)$ & $2(1.3 \%)$ \\
\hline & $40-59$ & $42(47.2 \%)$ & $74(22.6 \%)$ & $40(23.5 \%)$ & $34(21.5 \%)$ \\
\hline & $>60$ & $13(14.6 \%)$ & $252(76.8 \%)$ & $130(76.5 \%)$ & $122(77.2 \%)$ \\
\hline \multirow{2}{*}{$\begin{array}{l}\text { Age at diabetes } \\
\text { diagnostic }\end{array}$} & Mean & 22.9 & 48.6 & 52.0 & 44.9 \\
\hline & SD & 12.0 & 10.5 & 10.0 & 9.7 \\
\hline \multirow{3}{*}{ Insulin prescription } & At diagnostics & $69(77.5 \%)$ & $13(4.0 \%)$ & $2(1.2 \%)$ & $11(7.0 \%)$ \\
\hline & Within 3 months & $1(1.1 \%)$ & $1(0.3 \%)$ & $0(0.0 \%)$ & $1(0.6 \%)$ \\
\hline & No & $19(21.4 \%)$ & $314(95.7 \%)$ & $168(98.8 \%)$ & $146(92.4 \%)$ \\
\hline \multirow{2}{*}{ BMI } & Mean & 25.0 & 29.0 & 28.4 & 29.5 \\
\hline & SD & 3.6 & 4.7 & 4.3 & 4.9 \\
\hline \multicolumn{6}{|l|}{ Employment } \\
\hline \multicolumn{2}{|l|}{ work full-time } & $70 \%$ & $23 \%$ & $24 \%$ & $21 \%$ \\
\hline & $8 \%$ & $3 \%$ & $4 \%$ & $3 \%$ \\
\hline \multicolumn{2}{|l|}{ not working; looking } & $5 \%$ & $2 \%$ & $1 \%$ & $3 \%$ \\
\hline \multicolumn{2}{|c|}{ not working; not looking } & $0 \%$ & $6 \%$ & $8 \%$ & $4 \%$ \\
\hline \multicolumn{2}{|l|}{ unable to work } & $1 \%$ & $3 \%$ & $1 \%$ & $4 \%$ \\
\hline \multicolumn{2}{|l|}{ retired } & $13 \%$ & $63 \%$ & $61 \%$ & $65 \%$ \\
\hline \multicolumn{2}{|l|}{ student } & $3 \%$ & $0 \%$ & $0 \%$ & $0 \%$ \\
\hline \multicolumn{2}{|c|}{ stay at home spouse/partner } & $0 \%$ & $0 \%$ & $1 \%$ & $0 \%$ \\
\hline \multicolumn{6}{|c|}{ Monthly household income } \\
\hline \multicolumn{2}{|l|}{$€ 0-€ 505$} & $6 \%$ & $13 \%$ & $11 \%$ & $14 \%$ \\
\hline \multicolumn{2}{|l|}{$€ 506-€ 2000$} & $55 \%$ & $64 \%$ & $61 \%$ & $68 \%$ \\
\hline \multicolumn{2}{|l|}{$€ 2001-€ 5000$} & $25 \%$ & $15 \%$ & $17 \%$ & $14 \%$ \\
\hline \multicolumn{2}{|l|}{$€ 5001-€ 10000$} & $2 \%$ & $2 \%$ & $2 \%$ & $1 \%$ \\
\hline \multicolumn{2}{|l|}{$€ 10001-€ 20000$} & $1 \%$ & $0 \%$ & $0 \%$ & $0 \%$ \\
\hline \multicolumn{2}{|l|}{ declined to answer } & $11 \%$ & $6 \%$ & $9 \%$ & $3 \%$ \\
\hline \multicolumn{6}{|l|}{ Education } \\
\hline \multicolumn{2}{|c|}{ basic education $-1^{\text {st }}$ cycle } & $8 \%$ & $43 \%$ & $37 \%$ & $49 \%$ \\
\hline \multicolumn{2}{|c|}{ basic education $-2^{\text {nd }}$ cycle } & $7 \%$ & $7 \%$ & $7 \%$ & $7 \%$ \\
\hline \multicolumn{2}{|c|}{ basic education $-3^{\text {rd }}$ cycle } & $14 \%$ & $15 \%$ & $14 \%$ & $15 \%$ \\
\hline \multicolumn{2}{|l|}{ secondary education } & $27 \%$ & $18 \%$ & $21 \%$ & $15 \%$ \\
\hline \multicolumn{2}{|l|}{ bachelor's degree } & $9 \%$ & $4 \%$ & $4 \%$ & $3 \%$ \\
\hline master's degree & & $35 \%$ & $13 \%$ & $16 \%$ & $9 \%$ \\
\hline doctoral degree & & $1 \%$ & $0 \%$ & $1 \%$ & $0 \%$ \\
\hline no qualifications/edu & cation & $0 \%$ & $0 \%$ & $0 \%$ & $1 \%$ \\
\hline other & & $0 \%$ & $0 \%$ & $1 \%$ & $0 \%$ \\
\hline
\end{tabular}

BMI: body mass index (calculated as weight / square height)

about my weight", the agreement rating was $38.2 \%$ ( $n=$ $34)$ for type 1 and $51.1 \%(n=167)$ for type 2 patients. Both patient groups mostly agreed with the sentence "My family argues with me about how I choose to take care of my diabetes", with an agreement rating of $65.1 \%(n=58)$ for type 1 and $66.7 \%(n=218)$ for type 2 . Regarding discrimination, $22.5 \%(n=20)$ of type 1 and $4.9 \%$ of type 2 patients agreed with the sentence "I have been discriminated against because I have diabetes".

\section{Caring of diabetes: the role of family members}

The quality of life of FM was also evaluated using the WHOQOL-BREF scale and WHO-5. Most FMs of type 1 patients, $59.3 \%(n=19)$, classified their QoL as 'Good/Very 
Table 2 - PWD perceived quality of life

\begin{tabular}{llcccc}
\hline & & $\begin{array}{c}\text { Type 1 } \\
(n=89)\end{array}$ & $\begin{array}{c}\text { Type 2 } \\
(n=328)\end{array}$ & $\begin{array}{c}\text { Type 2 } \\
\text { non-insulin treated } \\
(n=170)\end{array}$ & $\begin{array}{c}\text { Type 2 } \\
\text { insulin treated } \\
(n=158)\end{array}$ \\
\hline \multirow{3}{*}{ WHOQOL-BREF } & Very Poor/Poor & $3(3.4 \%)$ & $25(7.6 \%)$ & $8(4.7 \%)$ & $17(10.8 \%)$ \\
& Neither poor nor good & $26(29.2 \%)$ & $159(48.5 \%)$ & $80(47.1 \%)$ & $79(50.0 \%)$ \\
PAID-5 & Good/Very Good & $60(67.4 \%)$ & $144(43.9 \%)$ & $82(48.2 \%)$ & $62(39.2 \%)$ \\
& Low distress & $25(28.1 \%)$ & $127(38.8 \%)$ & $83(49.1 \%)$ & $44(27.8 \%)$ \\
\hline
\end{tabular}

WHOQOL-BREF: abbreviated version of the World Health Organization quality of life assessment questionnaire WHQOL-100; PAID-5: Problem Areas in Diabetes scale - short form.

Table 3 - Impact of diabetes in PWD's QoL $(n=120)$

\begin{tabular}{|c|c|c|c|c|c|}
\hline & & $\begin{array}{l}\text { Slightly to very } \\
\text { negative impact }\end{array}$ & $\begin{array}{c}\text { No } \\
\text { impact }\end{array}$ & $\begin{array}{l}\text { Slightly to very } \\
\text { positive impact }\end{array}$ & $\begin{array}{c}\text { Not } \\
\text { applicable }\end{array}$ \\
\hline \multirow{2}{*}{ Physical health } & Type $1(n=89)$ & $56(62.9 \%)$ & $24(27.0 \%)$ & $9(10.1 \%)$ & $0(0.0 \%)$ \\
\hline & Type $2(n=328)$ & $159(48.5 \%)$ & $145(44.2 \%)$ & $24(7.3 \%)$ & $0(0.0 \%)$ \\
\hline \multirow{2}{*}{ Financial situation } & Type $1(n=89)$ & $34(38.2 \%)$ & $54(60.7 \%)$ & $1(1.1 \%)$ & $0(0.0 \%)$ \\
\hline & Type $2(n=328)$ & $120(36.6 \%)$ & $199(60.7 \%)$ & $9(2.7 \%)$ & $0(0.0 \%)$ \\
\hline \multirow{2}{*}{ RFFP } & Type $1(n=89)$ & $18(20.2 \%)$ & $62(69.7 \%)$ & $7(7.9 \%)$ & $2(2.2 \%)$ \\
\hline & Type $2(n=328)$ & $38(11.6 \%)$ & $273(83.2 \%)$ & $16(4.9 \%)$ & $1(0.3 \%)$ \\
\hline \multirow{2}{*}{ Leisure activities } & Type $1(n=89)$ & $29(32.6 \%)$ & $53(59.6 \%)$ & $6(6.7 \%)$ & $1(1.1 \%)$ \\
\hline & Type $2(n=328)$ & $61(18.6 \%)$ & $255(77.7 \%)$ & $9(2.7 \%)$ & $3(1.0 \%)$ \\
\hline \multirow{2}{*}{ Work or studies } & Type $1(n=89)$ & $24(27.0 \%)$ & $47(52.8 \%)$ & $3(3.4 \%)$ & $15(16.8 \%)$ \\
\hline & Type $2(n=328)$ & $17(5.2 \%)$ & $95(29.0 \%)$ & $3(0.9 \%)$ & $213(64.9 \%)$ \\
\hline \multirow{2}{*}{ Emotional well-being } & Type $1(n=89)$ & $50(56.1 \%)$ & $35(39.3 \%)$ & $4(4.6 \%)$ & $0(0.0 \%)$ \\
\hline & Type $2(n=328)$ & $139(41.9 \%)$ & $185(56.4 \%)$ & $4(1.2 \%)$ & $0(0.0 \%)$ \\
\hline
\end{tabular}

PWD: person with diabetes; QoL: quality of life; RFFP: relationships with family, friends and peers

Good', $39.5 \%(n=12)$ as 'Neither poor nor good' and $1.1 \%$ $(n=1)$ as 'Very Poor/Poor'. The majority of FMs of type 2 patients, $45.9 \%(n=42)$, classified their $\mathrm{QoL}$ as 'Neither poor nor good', $43.2 \%(n=39)$ as 'Good/Very Good', and $10.8 \%(n=10)$ as 'Very Poor/Poor'. Table 4 summarizes the impact of diabetes on FM's QoL in the same six dimensions analysed for PWD plus one: relationship with the PWD the FM lives with. Emotional well-being and physical health were reported as the areas that were most negatively impacted area in FM's QoL, for both types of DM patients.

FMs attitudes about PWD condition were also analysed. For the sentence "I am very worried about the risk of hypoglycaemic events", FMs of type 1 PWD reported an agreement rating of $66 \%$ and FMs of type 2 PWD, $46 \%$. Most FMs reported an agreement for the sentence "we argue about how PWD choses to take care of diabetes": $72 \%$ for FMs of type 1 PWD and $81 \%$ for FMs of type 2 PWD. Yet, most FMs reported an agreement for the sentence "PWD diabetes is currently well controlled: $56 \%$ for FMs of type 1 PWD and $79 \%$ for FMs of type 2 PWD.

When PWD were asked about if they inform FM about the best way to support their diabetes management, both $51.6 \%$ of type $1(n=46)$ and $16.2 \%$ of type 2 patients $(n=$ 250) reported that they never/rarely did it. Most FMs also pointed out that the PWD they lived with never/rarely did it: with $58.1 \%(n=50)$ for type 1 and $71.4 \%(n=25)$ for type 2 patients. Similar results were obtained for how often PWD ask for help in diabetes management: most type 1 PWD (54.0\%; $n=48)$, and type 2 PWD $(75.6 \% ; n=248)$ reported that they never/rarely do it. Most FMs also reported that they were never/rarely asked for help: $58.1 \%(n=50)$ for type 1 and $71.5 \%(n=25)$ for type 2 patients.

Considering diabetes care responsibility, FMs' answers showed that PWD are mainly responsible for it: remembering to take his/her medication (type $1-59.3 \% ; n=51$; type $2-74.3 \%$; $n=26$ ); measuring his/her blood sugar (type $1-81.4 \% ; n=70$; type $2-77.2 \% ; n=27$ ); injecting his/her medication (type $1-80.2 \% ; n=69$; type 2 - not assessed); and planning time for exercise or physical activity (type 1 $47.7 \% ; n=41$; type $2-48.6 \% ; n=17$ ). Still, FMs are the main responsible in planning and cooking healthy meals (type 1 - 34.9\%; $n=30$; type 2 - 37.2\%; $n=13$ ).

FMs reported high agreement scores on the following sentences: "you usually attend his/her visits to the HCPs regarding his/her diabetes" (type $1-82.4 \% ; n=52$; type 2 - 80.0\%; n = 28); "you wish the person you live with would take greater responsibility in caring for his/her diabetes" (type $1-60.4 \% ; n=70 ;$ type $2-48.5 \% ; n=17$ ); and "you are confident that the person you live with can manage his/ her diabetes without your help" (type $1-62.8 \% ; n=54$; type 2 - $54.2 \% ; n=19$ ).

Regarding supportive behaviours, PWD reported that FMs often/always warn them if they are not managing diabetes properly (type $1-60.0 \% ; n=39$; type $2-65.0 \%$; 
Table 4 - Impact of diabetes in FM's QoL $(n=120)$

\begin{tabular}{|c|c|c|c|c|c|}
\hline & & $\begin{array}{l}\text { Slightly to very } \\
\text { negative impact }\end{array}$ & $\begin{array}{c}\text { No } \\
\text { impact }\end{array}$ & $\begin{array}{l}\text { Slightly to very } \\
\text { positive impact }\end{array}$ & $\begin{array}{c}\text { Not } \\
\text { applicable }\end{array}$ \\
\hline \multirow{2}{*}{ Physical health } & Group $1(n=85)$ & $15(17.6 \%)$ & $58(68.2 \%)$ & $7(8.2 \%)$ & $5(5.9 \%)$ \\
\hline & Group $2(n=35)$ & $6(17.1 \%)$ & $26(74.2 \%)$ & $3(8.6 \%)$ & $0(0.0 \%)$ \\
\hline \multirow{2}{*}{ Financial situation } & Group $1(n=85)$ & $28(32.9 \%)$ & $52(61.1 \%)$ & $1(1.2 \%)$ & $4(4.7 \%)$ \\
\hline & Group $2(n=35)$ & $12(34.2 \%)$ & $225(62.9 \%)$ & $1(2.9 \%)$ & $0(0.0 \%)$ \\
\hline \multirow{2}{*}{ RFFP } & Group $1(n=85)$ & $10(11.8 \%)$ & $69(81.2 \%)$ & $4(4.7 \%)$ & $2(2.4 \%)$ \\
\hline & Group $2(n=35)$ & $3(8.6 \%)$ & $28(80.0 \%)$ & $3(8.6 \%)$ & $0(0.0 \%)$ \\
\hline \multirow{2}{*}{ Leisure activities } & Group $1(n=85)$ & $21(24.7 \%)$ & $56(65.9 \%)$ & $5(5.9 \%)$ & $3(3.5 \%)$ \\
\hline & Group $2(n=35)$ & $3(8.6 \%)$ & $29(82.9 \%)$ & $3(8.6 \%)$ & $0(0.0 \%)$ \\
\hline \multirow{2}{*}{ Work or studies } & Group $1(n=85)$ & $12(14.1 \%)$ & $32(37.6 \%)$ & $2(2.4 \%)$ & $39(45.9 \%)$ \\
\hline & Group $2(n=35)$ & $0(0.0 \%)$ & $11(31.4 \%)$ & $0(0.0 \%)$ & $24(68.6 \%)$ \\
\hline \multirow{2}{*}{ Emotional well-being } & Group $1(n=85)$ & $35(41.1 \%)$ & $44(51.8 \%)$ & $3(3.5 \%)$ & $3(3.5 \%)$ \\
\hline & Group $2(n=35)$ & $13(37.1 \%)$ & $21(60.0 \%)$ & $1(2.9 \%)$ & $0(0.0 \%)$ \\
\hline \multirow{2}{*}{ RPWD } & Group $1(n=85)$ & $19(22.4 \%)$ & $56(65.9 \%)$ & $6(7.1 \%)$ & $4(4.7 \%)$ \\
\hline & Group $2(n=35)$ & $8(22.9 \%)$ & $26(74.3 \%)$ & $1(2.9 \%)$ & $0(0.0 \%)$ \\
\hline
\end{tabular}

FM: family member; QoL: quality of life; RFFP: relationships with family, friends and peers; RPWD: relationship with the person with diabetes you live with

$\mathrm{n}=117$ ) or congratulate them otherwise (type $1-43.1 \% ; \mathrm{n}=$ 28; type 2 - 33.9\%; $n=61$ ). Likewise, FMs also reported the same supportive behaviour, often/always warning PWD if they believe he/she is not managing diabetes properly (type 1 - 50.6\%; n = 43; type $2-57.1 \% ; n=20$ ) or congratulating PWD otherwise (type $1-40.0 \%$; $n=34$; type $2-37.1 \%$; n = 13). Most of PWD also reported that FMs often/always acknowledge their difficulties in living with diabetes (type $1-76.9 \%$; $n=50$; type 2 - 69.4\%; $n=125$ ); in line with FMs' reported perception that they take into consideration the difficulties shared by the PWD they live with (type 1 - 66.7\%; n = 56; type $2-65.8 \% ; n=23$ ).

\section{Caring of diabetes: the perspective of health care pro- fessionals}

Table 5 summarizes the data concerning the HCPs beliefs about diabetes management in three dimensions: (i) HCP understanding and management of patient emotions, (ii) HCP influence over patient management of the disease and (iii) HCP role in patient advocacy. Overall, the three groups of HCPs recognized the importance of the 3 dimensions analysed.

Regarding PACIC results for HCPs, $35.0 \%(n=14)$ hospital-based specialists, 20.6\% $(n=14) \mathrm{PCPs} / \mathrm{GPs} / \mathrm{FPs}$, and $27.7 \%(n=34)$ NODs stated that they ask their pa- tients most of the time or always how diabetes affects their life. Concerning the question "Do you ask your patients for ideas when making a diabetes care plan", the agreement scores (most of the times/always) were: $55.0 \%(n=22)$ for hospital-based specialists; $50.0 \%(\mathrm{n}=354)$ for PCPs/GPs / FPs; and 43.9\% ( $n=54$ ) for NODs. For the question, "Do you encourage your patients to ask questions", the agreement scores (most of the times/always) were: $77.5 \%$ ( $n=$ 31 ) for hospital based specialists; $75.0 \%(n=51)$ for PCPs/ GPs /FPs; and 65.8\% ( $\mathrm{n}=81$ ) for NODs.

Regarding PACIC results for PWD, 20.4\% ( $n=17)$ type 1 and $11.8 \%(n=36)$ Type 2 patients reported that they were asked most of the time or always about how diabetes affects their life in the past 12 months. For the statement "I was helped to make plans to achieve my diabetes care goals", agreement scores (most of the time/always) were $63.8 \%(n=53)$ for type 1 and 38.8\% ( $n=120)$ for type 2 patients. For the statement "I was helped to make plans for how to get support from friends, family or community", agreement scores (most of the time/always) were $20.4 \%$ (n $=17)$ for type 1 and $11.4 \%(n=35)$ for type 2 patients. For the statement "I am satisfied that my care is well organized", agreement scores (most of the time/always) were $57.3 \%$ (n $=47)$ for type 1 and $48.7 \%(n=149)$ for type 2 patients.

Table 5 - HCPs' beliefs about diabetes management, rating of " 5 " or "6" on a 6-point agreement scale $(n=227)$

\begin{tabular}{lccc}
\hline & $\begin{array}{c}\text { PCPs / GPs / FPs } \\
(\mathrm{n}=68)\end{array}$ & $\begin{array}{c}\text { Hospital specialists* } \\
(\mathrm{n}=40)\end{array}$ & $25(62.5 \%)$ \\
\hline $\begin{array}{l}\text { My success in caring for people with diabetes depends largely on my } \\
\text { ability to understand and manage their emotional issues. }\end{array}$ & $23(33.8 \%)$ & $3(7.5 \%)$ & $48(40.3 \%)$ \\
$\begin{array}{l}\text { HCPs have a very limited influence on how well people take care of } \\
\text { their diabetes. }\end{array}$ & $5(7.4 \%)$ & $23(57.5 \%)$ \\
$\begin{array}{l}\text { It is important for me to advocate on behalf of PWD and be involved in } \\
\text { health policy issues for improvement diabetes care. }\end{array}$ & $32(47.1 \%)$ & $46(38.7 \%)$ \\
\hline
\end{tabular}

*: Endocrinologists and internal medicine specialists

HCP: healthcare professional; PCP, primary care physician; GP: general practitioner; FP: family physician; NDO: nurses, dietitian and other healthcare professionals. 
Table 6 - PWD, FMs and HCPs' beliefs about areas for improvement

\begin{tabular}{llccccc}
\hline & $\begin{array}{c}\text { Acceptance } \\
\text { of people with } \\
\text { diabetes as equal } \\
\text { members of society }\end{array}$ & $\begin{array}{c}\text { Convenient and } \\
\text { safe places to } \\
\text { participate in } \\
\text { physical activity }\end{array}$ & $\begin{array}{c}\text { Places to buy } \\
\text { healthy and } \\
\text { affordable food }\end{array}$ & $\begin{array}{c}\text { Workplaces which } \\
\text { it easy for people } \\
\text { to manage their } \\
\text { diabetes }\end{array}$ & $\begin{array}{c}\text { Earlier diagnosis } \\
\text { and treatment of } \\
\text { diabetes }\end{array}$ \\
\hline \multirow{2}{*}{ PWD } & Type 1 $(n=89)$ & $49(55.0 \%)$ & $56(62.9 \%)$ & $74(83.1 \%)$ & $58(65.2 \%)$ & $82(92.1 \%)$ \\
& Type 2 $(n=328)$ & $210(64.0 \%)$ & $236(72.0 \%)$ & $272(82.9 \%)$ & $262(79.9 \%)$ & $302(92.0 \%)$ \\
FMs & Group 1 $(n=85)$ & $51(60.0 \%)$ & $64(75.3 \%)$ & $70(82.3 \%)$ & $64(75.3 \%)$ & $78(91.8 \%)$ \\
& Group 2 $(n=35)$ & $18(51.4 \%)$ & $25(71.4 \%)$ & $30(85.7 \%)$ & $23(65.7 \%)$ & $31(91.4 \%)$ \\
HCPs & SpPs/GPs $(n=68)$ & $15(22.1 \%)$ & $32(47.1 \%)$ & $40(58.8 \%)$ & $26(38.2 \%)$ & $37(54.4 \%)$ \\
& Specialists $(n=40)$ & $13(32.5 \%)$ & $17(42.5 \%)$ & $18(45.0 \%)$ & $16(40.0 \%)$ & $23(57.5 \%)$ \\
\hline
\end{tabular}

PWD: person with diabetes; FM: family member; HCP: health care professional

\section{Areas of improvement}

The three study groups (PWD, FM and HCPs) were asked for their opinion about areas where they feel there was still a need for additional education to help people with diabetes in their community and society (Table 6). Earlier diagnosis and treatment of diabetes was the major area of concern for PWD and FMs - PWD type $1-92.1 \%(n=82)$, PWD type $2-92.0 \%(n=302)$; FMs type $1-91.8 \%(n=$ 78), FMs type $2-91.4 \%(n=31)$ - whereas for HCPs was a less relevant area of concern - PCPs/GPs /FPs- $54.4 \%$ ( $n=37)$; Hospital based specialists $-57.5 \%(n=23)$; NDOs $-53.8 \%(n=64)$. The same pattern of responses between PWD/FMs and HCPs was observed for all other areas for improvement assessed, with PWD/FMs reporting more concern (51\% - 83\% range of positive answers) and HCPs less concern ( $21 \%-59 \%$ range of positive answers). The main areas for improvement identified by HCPs were: "Availability of diabetes self-management education" [PCPs/GPs $67.6 \%(n=46)$; Hospital based specialists - 60.0\% ( $n=24)$; NDOs $-54.6 \%(n=65)$ ]; "Availability of resources for psychological support for diabetes" [PCPs/GPs/FPs - 61.8\% (n $=42$ ); Hospital based specialists - 65.0\% ( $n=26)$; NDOs $54.6 \%(n=65)$ ]; and "Planning and coordination of care for patients with multiple diseases" [PCPs/GPs/FPs $-60.3 \%$ (n $=41)$; Hospital based specialists - 45\% ( $n=18)$; NDOs $57.1 \%(n=68)]$.

\section{DISCUSSION}

In this study we have applied the DAWN2 protocol $^{10}$ to the Portuguese setting, addressing the wishes, needs, and attitudes of Portuguese diabetes patients, their relatives, and health professionals regarding the disease for the first time. The results obtained indicate that diabetes is associated with great physical and psychological burden for people with diabetes (PWD), with a negative impact on their physical health and emotional wellbeing. The psychological burden of diabetes was also clearly recognized in the original multinational DAWN2 study. ${ }^{13}$ Also in line with the DAWN2 multinational study, ${ }^{11,14}$ PWD in Portugal also reported high levels of diabetes-related distress. PWD's quality of life is affected by several factors which are associated with impaired disease self-management, and consequently, a gradual worsening of symptoms and disease-associated complications. ${ }^{15,16}$ We believe that in order to mitigate this deterioration of PWD quality of life it is important to invest on continuous follow-up and evaluation of patients, with a particular focus on their psychological health.

There are few studies in the literature addressing the perceptions of family members of PWD. Nevertheless, the key role of FMs on disease management and support has been extensively highlighted by the data collected in the original DAWN2 multinational study. ${ }^{11}$ In line with the original DAWN2 multinational study, in this study most PWD have recognized that their FMs often/always warn them if they are not managing diabetes properly and also take into consideration their difficulties in living with diabetes. Also in line with the original DAWN2 multinational study, ${ }^{11}$ this study showed that diabetes is often a psychological burden for FMs, with a significant impact on their emotional wellbeing, due to their concerns and relevant role in caring for their relatives. Interestingly this burden for FMs may not be recognized by PWD. In fact, according to the DAWN2 USA data, PWD perceived support to be less frequent and less helpful than FMs. ${ }^{17}$

Regarding the perceptions of HCPs, most HCPs in this study recognized they have a significant role in influencing PWD in terms of the disease self-management. In addition, most endocrinologists and internal medicine specialists recognize the importance of addressing the emotional issues of the patient and believe they have an active role in terms of PWD advocacy. Patient perceptions can be inconsistent with those from healthcare professionals. Interestingly, both groups in this study recognized a mutual cooperative attitude in managing the disease. In fact, most patients have reported receiving help from $\mathrm{HCPs}$ in making plans to achieve their diabetes care goals, while most HCPs agreed that they frequently ask their patients for ideas, when making a plan for their diabetes care. In contrast with the observations of the DAWN2 multinational study, ${ }^{18}$ Portuguese HCPs did not report being insufficiently prepared to provide diabetes self-management education to their patients.

In this study, we have also addressed the perceptions of PWD, FMs and HCPs concerning the organization of diabetes healthcare. PWD have reported to be generally satisfied 
with the organization of their healthcare. However, several areas of improvement were mentioned, namely an earlier diagnosis and treatment of the disease, which implies an improvement of healthcare access, expansion of diabetes screening, and more health literacy programs, as previously discussed based on the data from DAWN2 study..$^{13}$ In addition, in our study PWD and FMs recognized that there is still some discrimination in society against the disease. Once again, we believe this discrimination is a consequence of inefficient literacy campaigns, which translates into low acceptance levels. Finally, PWD and FMs also mentioned logistic areas of improvement, namely: lack of places to buy healthy and affordable food, adapting workplaces to the disease management, and lack of safe places to make physical activity. According to HCPs, accessibility to diabetes self-management education programs was reported as an area for improvement, which is quite relevant, since, as previously described, ${ }^{19}$ this kind of programs enables patients to gain important skills in managing their disease and live a healthier lifestyle, which translates into better clinical outcomes.

The DAWN2 study protocol has some limitations, namely concerning the difficulty to represent the DM population accurately, as previously recognized. ${ }^{10}$ In particular, a great majority of PWD and FMs in this study were enrolled in the APDP headquarters, which mostly serves the Lisbon metropolitan area, the most populated area in Portugal, but still introducing a bias in the representativeness of our sample. In addition, we had difficulty in enrolling HCPs, particularly physicians, and to collect enough valid answers, as this group was not supported by an interviewer and, instead, self-administered the questionnaires. Our overall recruitment rate was lower than what is recommended by the DAWN2 protocol (767 vs 900 participants per country). Nevertheless, we managed to respect the recommended PWD:FM:HCP ratios: $54 \%: 16 \%: 30 \%$ in this study vs 55\%:13\%:31\% in DAWN2 protocol.

Despite the DAWN2 study limitations, the generated multinational data was very useful to benchmark against different countries, enabling the identification of best practices and detection of areas for improvement. ${ }^{20}$

\section{CONCLUSION}

In this study, we have used for the first time in Portugal used the DAWN2 protocol to address the wishes, needs, and attitudes of Portuguese diabetes patients, their relatives, and healthcare professionals regarding the disease. The collected data suggest that diabetes has a negative impact on the physical health and emotional well-being of patients in Portugal, and is also a psychosocial burden for family members. In addition, this study has identified as main areas of improvement the earlier diagnosis and treatment of diabetes and diabetes self-management education.
Altogether, the insights obtained with this study can guide the re-definition of priorities and pave the way for the design of better integrated healthcare strategies for diabetes management, reducing the physical and psychological burden of people living with diabetes.

\section{ACKNOWLEDGEMENTS}

The authors thank all persons with diabetes, family members, informal carers and health-care professionals who have kindly accepted to participate in this study. This study was supported by Novo Nordisk. In the preparation of this article, the authors had the collaboration of medical writer Duarte Oliveira (W4Research) financially supported by Novo Nordisk. Novo Nordisk had no role in the preparation of the manuscript or in the decision to submit the manuscript for publication.

\section{AUTHORS CONTRIBUTION}

DMO: Data analysis and interpretation. definition of the design and structure of the article. Written document development and revisions.

LS, RTR, AC: Data analysis and interpretation; critical review of the manuscript. Approval of the final version. Agreement with all the developed topics, fidelity and precision of the delivered results.

SS, JMB, JFR: Significant contribution for the concept of the work and review of the paper structure. Approval of the final version. Agreement with all the developed topics, fidelity and precision of the delivered results.

MJA: Significant contribution as a nutritional educator in the analysis and interpretation of data. Approval of the final version. Agreement with all the developed topics, fidelity and precision of the delivered results.

\section{PROTECTION OF HUMANS AND ANIMALS}

The authors declare that the procedures were followed according to the regulations established by the Clinical Research and Ethics Committee and to the Helsinki Declaration of the World Medical Association updated in 2013.

\section{DATA CONFIDENTIALITY}

The authors declare having followed the protocols in use at their working center regarding patients' data publication.

\section{COMPETING INTERESTS}

The authors have declared that no competing interests exist.

\section{FUNDING SOURCES}

This research received no specific grant from any funding agency in the public, commercial, or not-for-profit sectors.

\section{REFERENCES}

1. American Diabetes Association. Introduction: standards of medical care in diabetes-2018. Diabetes Care. 2018;41:S1-2.
2. Koetsenruijter J, van Lieshout J, Vassilev I, Portillo MC, Serrano M, Knutsen I, et al. Social support systems as determinants of self- 
management and quality of life of people with diabetes across Europe: study protocol for an observational study. Health Qual Life Outcomes. 2014;12:29.

3. World Health Organization. Global report on diabetes. Geneva: WHO; 2016.

4. Nielsen HB, Ovesen LL, Mortensen LH, Lau CJ, Joensen LE. Type 1 diabetes, quality of life, occupational status and education level - a comparative population-based study. Diabetes Res Clin Pract. 2016;121:62-8.

5. Ausili D, Bulgheroni M, Ballatore P, Specchia C, Ajdini A, Bezze S, et al. Self-care, quality of life and clinical outcomes of type 2 diabetes patients: an observational cross-sectional study. Acta Diabetol. 2017;54:1001-8.

6. Carolan M, Holman J, Ferrari M. Experiences of diabetes selfmanagement: a focus group study among Australians with type 2 diabetes. J Clin Nurs. 2015;24:1011-23.

7. Baig AA, BenitezA, Quinn MT, Burnet DL. Family interventions to improve diabetes outcomes for adults. Ann N Y Acad Sci. 2015;1353:89-112.

8. Rintala TM, Jaatinen P, Paavilainen E, Astedt-Kurki P. Interrelation between adult persons with diabetes and their family: a systematic review of the literature. J Fam Nurs. 2013;19:3-28.

9. Goetz K, Szecsenyi J, Campbell S, Rosemann T, Rueter G, Raum E, et al. The importance of social support for people with type 2 diabetes - a qualitative study with general practitioners, practice nurses and patients. Psychosoc Med. 2012;9:Doc02.

10. Peyrot M, Burns KK, Davies M, Forbes A, Hermanns N, Holt R, et al. Diabetes Attitudes Wishes and Needs 2 (DAWN2): a multinational, multi-stakeholder study of psychosocial issues in diabetes and personcentred diabetes care. Diabetes Res Clin Pract. 2013 Feb;99(2):17484.

11. Nicolucci A, Kovacs Burns K, Holt RIG, Comaschi M, Hermanns N, Ishii $\mathrm{H}$, et al. Diabetes Attitudes, Wishes and Needs second study (DAWN2 ${ }^{\mathrm{TM}}$ ): Cross-national benchmarking of diabetes-related psychosocial outcomes for people with diabetes. Diabet Med. 2013;30:767-77.

12. Peyrot M, Rubin RR, Lauritzen T, Snoek FJ, Matthews DR, Skovlund SE.
Psychosocial problems and barriers to improved diabetes management: results of the Cross-National Diabetes Attitudes, Wishes and Needs (DAWN) Study. Diabet Med. 2005;22:1379-85.

13. Speight J. DAWN2 shines more light on the psychological burden of living with diabetes and on the correlates of quality psychological care. Diabet Med. 2016;33:1172-3.

14. Kovacs Burns K, Nicolucci A, Holt RIG, Willaing I, Hermanns N, Kalra S et al. Diabetes Attitudes, Wishes and Needs second study (DAWN2 ${ }^{\mathrm{TM}}$ ): cross-national benchmarking indicators for family members living with people with diabetes. Diabet Med. 2013;30:778-88.

15. Mosnier-Pudar H, Hochberg G, Eschwege E, Halimi S, Virally ML, Guillausseau PJ, et al. How patients' attitudes and opinions influence self-care behaviours in type 2 diabetes. Insights from the French DIABASIS Survey. Diabetes Metab. 2010;36:476-83.

16. Zagarins SE, Allen NA, Garb JL, Welch G. Improvement in glycemic control following a diabetes education intervention is associated with change in diabetes distress but not change in depressive symptoms. J Behav Med. 2012;35:299-304.

17. Peyrot M, Egede L, Funnell M, Hsu W, Ruggiero L, Stuckey H. US ethnic group differences in family member support for people with diabetes in the 2nd Diabetes Attitudes, Wishes and Needs (DAWN2) Study. Diabetes Educ. 2018;44:249-59.

18. Byrne JL, Davies MJ, Willaing I, Holt RI, Carey ME, Daly H, et al. Deficiencies in postgraduate training for healthcare professionals who provide diabetes education and support: results from the Diabetes Attitudes, Wishes and Needs (DAWN2) study. Diabet Med. 2017;34:1074-83.

19. Galindo Rubio M, Jansà Morató $M$, Menéndez Torre E. Therapeutic education and self-care: Results from the cross-sectional study Diabetes, Attitudes, Wishes and Needs 2 (DAWN2) in Spain. Endocrinol y Nutr. 2015;62:391-9.

20. Tarkun İ, Dumanli Özgöksu S. Attitudes, wishes, and needs of diabetes patients and their relatives: Turkish data from the DAWN2 study. Turkish J Med Sci. 2017;47:447-54. 